\title{
Transport mechanisms of airborne particulate matters in partitioned indoor environment
}

\author{
Tsang-Jung Chang*, Ting-Shing $\mathrm{Hu}$ \\ Department of Bioenvironmental Systems Engineering, National Taiwan University, Taipei 10617, Taiwan, ROC
}

Received 3 August 2006; received in revised form 20 November 2006; accepted 10 January 2007

\begin{abstract}
The main objective of this study is to investigate the transport mechanisms of size-dependent airborne particulate matters in partitioned indoor environment. A three-dimensional Lagrangian particle tracking model is developed herein and validated by reliable experimental measurement. Four major particle-driving mechanisms (the gravitational force, the drag force, the Brownian motion force and the Saffman lift force) are considered in the model. Five kinds of particle transport mechanism scenarios are performed, including the dynamic equation scenario (all considered), the Brownian-motion-neglected scenario, the drag-force-neglected scenario, the lift-forceneglected scenario, and the inertial-force-neglected scenario (neglecting both the drag force and lift force). Seven different particle aerodynamic diameters $(10,5,2.5,1,0.5,0.1$ and $0.05 \mu \mathrm{m})$, ranging from coarse to ultra-fine particle ranges, are used to investigate the relationship between particle size and each transport mechanism scenario. The results show that the influence of the drag force and the inertial force is significant for particle diameter larger than $1 \mu \mathrm{m}$, and the Brownian motion force is important for particle diameter smaller than $0.5 \mu \mathrm{m}$. The Saffman lift force cannot be neglected in a specific range of particle sizes between 2.5 and $5 \mu \mathrm{m}$.
\end{abstract}

(C) 2007 Elsevier Ltd. All rights reserved.

Keywords: Particle transport mechanism; Particulate matter; Partitioned environment

\section{Introduction}

Airborne particle matters (PM) are ubiquitous in indoor environment, which could cause adverse impact on the quality of life and human health [1,2]. Therefore, better understanding of the transport behaviors of PM is considered to be valuable for assessing the hazards of indoor environment. Studies of indoor PM transport behavior have been conducted by numerical simulations and experimental measurements [3,4]. Generally, experimental measurements can provide useful details of PM transport behavior and airflow patterns only on limited number of sample points, because it is very expensive to simultaneously obtain temporal and spatial information on airflow velocity distributions, PM size and concentration distributions for the entire buildings. Computational fluid dynamics (CFD) solutions are thus more adequate and convenient for analyzing detail PM concentration distribu-

\footnotetext{
${ }^{*}$ Corresponding author. Tel.: + 886223622977 ; fax: +886223635854 .

E-mail address: tjchang@ntu.edu.tw (T.-J. Chang).
}

tions inside a building, because building configurations can be numerically made and modified as required within a short time. Numerical simulations of PM transport can be classified into the Eulerian and Lagrangian approaches. Most Eulerian approach works [5-8] are mainly based on the advection-diffusion equation together with the gravity settling, which takes less computing resources. However, this approach models the particulate phase as a continuum phase, and is only adequate for simulating gaseous pollutants or small, noninertial particles that exactly follow indoor airflow.

Since 1996, the continuing increase in the speed of computers makes numerical simulation more applicable. More and more CFD researches [9-16] have been using the Lagrangian approach to numerically examine indoor PM transport behaviors. All of the aforementioned Lagrangian works adapt the particle dynamic equation [12-16] or its simplified form [9-11]. The particle dynamic equation considers four major particle driving mechanisms, i.e., the gravitational force, $F_{\mathrm{G}}$, the drag force, $F_{\mathrm{D}}$, the Saffman lift force, $F_{\mathrm{S}}$, and the Brownian motion force, $F_{\mathrm{B}}$, and can be 


\begin{tabular}{|c|c|c|c|}
\hline \multicolumn{2}{|c|}{ Nomenclature } & $\left\langle\bar{u}_{i}\right\rangle$ & time-averaged fluid velocity in the $x_{i}$ direction \\
\hline$C$ & particle mass concentration & $\bar{u}_{i}^{\prime}$ & turbulent component of the instantaneous fluid \\
\hline$C_{\mathrm{s}}$ & Smagorinsky constant & & velocity in the $x_{i}$ direction \\
\hline$d_{i j}$ & deformation rate tensor & $u_{i}^{\mathrm{p}}$ & particle velocity in the $x_{i}$ direction \\
\hline$d_{\mathrm{p}}$ & particle diameter & V & volume of each room \\
\hline$F_{\mathrm{B}}$ & Brownian motion force & $x_{i}$ & Cartesian coordinate \\
\hline$F_{\mathrm{D}}$ & drag force & $\rho$ & fluid density \\
\hline$F_{\mathrm{G}}$ & gravitational force & $\rho_{\mathrm{p}}$ & particle density \\
\hline$F_{\mathrm{S}}$ & Saffman lift force & $v$ & fluid kinematic viscosity \\
\hline$g$ & gravitational acceleration & $\tau_{i j}$ & subgrid-scale Reynolds stresses \\
\hline$G_{i}$ & $\begin{array}{l}\text { zero-mean, unit variance Gaussian random } \\
\text { number }\end{array}$ & $\begin{array}{l}\tau \\
\delta\end{array}$ & $\begin{array}{l}\text { relaxation time of the particle } \\
\text { unit delta function }\end{array}$ \\
\hline$M s$ & total mass of suspended particles & $v_{\text {sgs }}$ & sub-grid eddy viscosity \\
\hline $\bar{P}$ & fluid pressure & $\Delta$ & filtered grid size \\
\hline$S_{0}$ & spectral intensity & $\Delta S(t)$ & average deviation of displacement during the \\
\hline$\underset{\sim}{S}$ & density ratio between particle and adjacent fluid & & tracking period \\
\hline$S_{i j}$ & fluid strain rate & $\Delta t$ & time step \\
\hline
\end{tabular}

expressed as the following [17-19]:

$$
\begin{aligned}
\frac{1}{6} \pi \rho_{\mathrm{p}} d_{\mathrm{p}}^{3} \frac{\mathrm{d} u_{i}^{\mathrm{p}}}{\mathrm{d} t}= & F_{\mathrm{G}}+F_{\mathrm{D}}+F_{\mathrm{S}}+F_{\mathrm{B}} \\
= & \frac{1}{6} \pi d_{\mathrm{p}}^{3}\left(\rho_{\mathrm{p}}-\rho\right) g_{i} \delta_{i 3} \\
& -\frac{1}{6} \pi \rho_{\mathrm{p}} d_{\mathrm{p}}^{3} \frac{1}{\tau}\left(u_{i}^{\mathrm{p}}-\bar{u}_{i}\right) \\
& -\frac{1}{6} \pi \rho_{\mathrm{p}} d_{\mathrm{p}}^{3} \frac{5.188 v^{1 / 2} d_{i j}}{S d_{\mathrm{p}}\left(d_{l k} d_{k l}\right)^{1 / 4}}\left(u_{j}^{\mathrm{p}}-\bar{u}_{j}\right) \\
& +\frac{1}{6} \pi \rho_{\mathrm{p}} d_{\mathrm{p}}^{3} G_{i} \sqrt{\frac{\pi S_{0}}{\Delta t}}
\end{aligned}
$$

and

$$
\frac{\mathrm{d} x_{i}}{\mathrm{~d} t}=u_{i}^{\mathrm{p}},
$$

where $\bar{u}_{i}$ is the instantaneous fluid velocity, $u_{i}^{\mathrm{p}}$ the particle velocity, $\rho$ the fluid density, $\rho_{\mathrm{p}}$ the particle density, $x_{i}$ the coordinate of particles, $t$ the time, $d_{\mathrm{p}}$ the particle diameter, $S$ the density ratio between particle and adjacent fluid, $v$ the kinematic viscosity, $\delta$ the unit delta function, $\tau$ the relaxation time of the particle, and $d_{i j}\left(=\left(\bar{u}_{i, j}+\bar{u}_{j, i}\right) / 2\right)$ is the deformation rate tensor. The Brownian motion force is usually modeled as the Gaussian white noise random process, in which $G_{i}$ is zero-mean, unit variance independent Gaussian random number, $\Delta t$ is the time step, and $S_{0}$ is spectral intensity of the Gaussian white noise [17,19].

In Eq. (1), the significance of each mechanism acting on indoor PM transport depends on particle property (particle diameter and particle density), fluid property (kinematic viscosity and fluid density) and indoor airflow pattern (air velocity and pressure). Particle property and fluid property are usually characterized by particle aerodynamic diameter at the standard condition $\left(20^{\circ} \mathrm{C}, 1 \mathrm{~atm}\right)$ [4]. So far, only Hinds [4] has given the relative importance between the
Brownian motion force and the gravitational force in still air for various aerodynamic diameters at the standard condition, by comparing the analytical solutions of the Stokes-Einstein equation and the Stokes settling equation. However, most of the buildings worldwide use indoor partitions to separate the entire building into several isolate spaces, and have internal airflow between each space. The PM transport mechanisms in nonquiescent partitioned environment are quite different from those in still-air environment $[3,4,15]$. To be closer to the real situation, it would be more preferable to study the effect of different driving mechanisms on particle transport behaviors in nonquiescent partitioned environment for various particle aerodynamic diameters. Therefore, in the present study, a 3-D turbulent flow model and a Lagrangian particletracking model are built to understand particle transport behaviors in partitioned environment. Particle concentrations and trajectories are calculated. A wide range of particle aerodynamic diameters, from coarse to ultra fine particle size ranges, are used to investigate the relationship between aerodynamic diameters and particle driving mechanisms.

\section{Numerical model}

For investigating the PM transport mechanisms in partitioned indoor environment, two numerical models are adopted herein, namely the Eulerian indoor airflow model and the Lagrangian particle trajectory-tracking model. First, the Eulerian indoor airflow model, conducting the large eddy simulation (LES) of the Eulerian turbulent flows indoors, is solved first to obtain the velocity and pressure distributions in the building. Next, the result of the velocity distribution is then input into the Lagranigan particle tracking model to calculate the 
velocities and trajectories of individual particles. These two models are described in the following.

\subsection{Three-dimensional (3-D) Eulerian indoor airflow model}

LES has recently received more and more attention and has been successfully applied to several natural ventilation problems $[3,12]$. A fully developed turbulent flow contains eddies of many length scales. In the LES procedure, small eddies are filtered out, and large eddies are solved numerically by computing the filtered equations. The influence of small eddies that are filtered out cannot be neglected and leads to the subgrid-scale Reynolds stress. The filtered continuity equation and momentum equation in LES are listed below

$\frac{\partial \bar{u}_{i}}{\partial x_{i}}=0$

and

$\frac{\partial \bar{u}_{i}}{\partial t}+\frac{\partial \bar{u}_{i} \bar{u}_{j}}{\partial x j}=-\frac{1}{\rho} \frac{\partial \bar{P}}{\partial x_{i}}+v \frac{\partial^{2} \bar{u}_{i}}{\partial x_{j} \partial x_{j}}-\frac{\partial \tau_{i j}}{\partial x_{j}}$

where $\bar{u}_{i}$ is the component of instantaneous fluid velocity in the $x_{i}$ direction, $\bar{P}$ the fluid pressure, and $\tau_{i j}$ represents the subgrid-scale Reynolds stresses, which is responsible for momentum exchanges between the subgrid-scale eddies and filtered-scale eddies. The bar "_" represents spatial

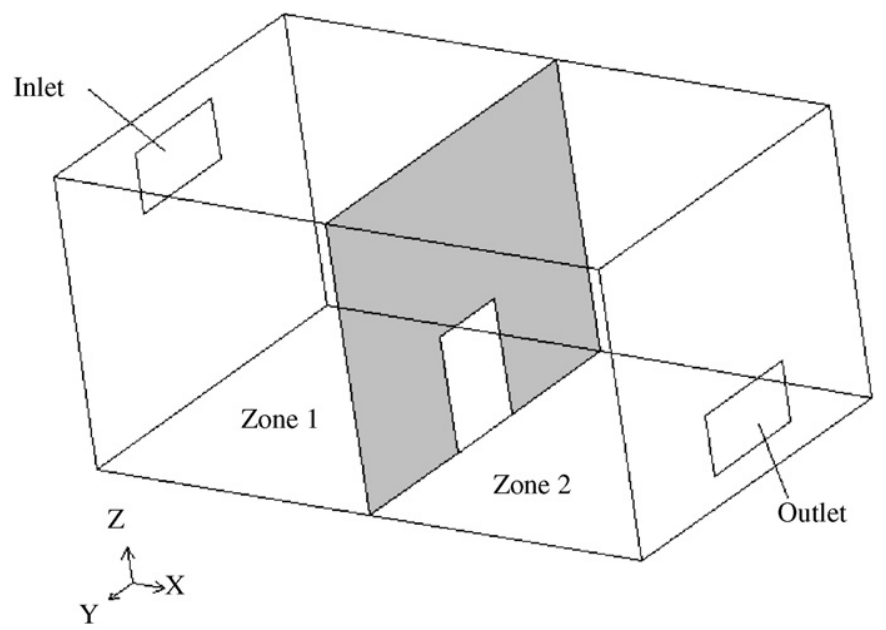

Fig. 1. Geometry of the two-zone building experiment (Lu et al., 1999). filtering. It should be pointed out that since the above LES procedure produces time-dependent solutions, the timeaveraged velocities $\left\langle\bar{u}_{i}\right\rangle$ can be obtained by statistically analyzing the instantaneous velocities $\bar{u}_{i}$ according to $\bar{u}_{i}=$ $\left\langle\bar{u}_{i}\right\rangle+\bar{u}_{i}^{\prime}$ in which $\bar{u}_{i}^{\prime}$ means the turbulent components of the instantaneous velocities. The details of the statistical analysis of LES solutions can be found in Chang et al. [15].

To simulate indoor airflow pattern in nonquiescent partitioned environment, the Smagorinsky subgrid-scale model together with the wall function model near the solid boundary is adopted. The Smagorinsky subgrid-scale model is related with fluid strain rate and can be expressed as

$\tau_{i j}=-2 v_{\mathrm{sgs}} \tilde{S}_{i j}$,

where $v_{\text {sgs }}$ is the sub-grid eddy viscosity $\left(=\left(C_{\mathrm{s}} \Delta\right)^{2}\left(2 \tilde{S}_{i j} \tilde{S}_{i j}\right)^{1 / 2}\right)$, $\tilde{S}_{i j}$ is the fluid strain rate, $\Delta=\left(\Delta_{x i} \Delta_{x j} \Delta_{x k}\right)^{1 / 3}$ the filtered grid width in three dimensions. $C_{\mathrm{s}}$, the Smagorinsky constant and usually between 0.1 and 0.2 [6,12], is 0.15 in the present study. The numerical details of solving Eqs. (3)-(5) can be found in Chang et al. [15].

\subsection{Three-dimensional Lagrangian particle tracking model}

The 3-D Lagrangian approach used herein is to track each individual particle through the airflow field by solving the particle dynamic equations (Eqs. (1) and (2)). Eqs. (1) and (2) are solved by the fourth-order predictor-corrector method [20]. Two kinds of boundary conditions are used in the present study. Trap boundary means that once a particle touches it, the particle is trapped, and particletracking process would cease. In the particle-tracking process, walls, floors and ceilings in the building are set as the trap boundaries. Outflow boundary is used for all the building exterior outlets. When a particle passes through the outflow boundary, the particle tracking is terminated.

Using Eqs. (1) and (2) in the present study, several assumptions are made to simplify the simulation of PM transport process without losing its accuracy. The size of particles is defined by the aerodynamic diameter of particles. Airborne particles have no influence on surrounding airflow field. There is no heat and mass transfer between particles and air. In the transport process, particle

Table 1

Particle parameters used in the model verification

\begin{tabular}{lllllll}
\hline Case no. & $\begin{array}{l}\text { Internal opening } \\
\text { size }(\mathrm{m} \times \mathrm{m})\end{array}$ & $\begin{array}{l}\text { Air change rate } \\
\left(\mathrm{h}^{-1}\right)\end{array}$ & Reynolds number & $\begin{array}{l}\text { No. of sample } \\
\text { particles }\end{array}$ & $\begin{array}{l}\text { Total initial } \\
\text { particle mass }(\mu \mathrm{g})\end{array}$ & $\begin{array}{l}\text { Particle mass carried by } \\
\text { each sample particle }(\mu \mathrm{g})\end{array}$ \\
\hline Case 1 & $0.70 \times 0.20$ & 11.48 & 1300 & 2400 & 152,857 & 63.69 \\
Case 2 & $0.70 \times 0.20$ & 14.00 & 1600 & 2400 & 104,695 & 43.62 \\
Case 3 & $0.70 \times 0.30$ & 11.57 & 1300 & 2400 & 119,520 & 49.80 \\
Case 4 & $0.70 \times 0.30$ & 12.71 & 1400 & 2400 & 94,536 & 39.39 \\
Case 5 & $0.70 \times 0.95$ & 9.22 & 1000 & 2400 & 117,880 & 49.12 \\
Case 6 & $0.70 \times 0.95$ & 10.26 & 1100 & 2400 & 147,664 & 61.53 \\
\hline
\end{tabular}


coagulation, electrostatic force, and phase change are not considered. Particle re-suspension is not expected because of the relatively low velocity of indoor environment.

\subsection{Particle mass concentration}

The concept of particle mass carried by each sample particle originally proposed by $\mathrm{Lu}$ et al. is introduced herein to calculate the PM mass concentration [9]. The particle mass carried by each sample particle is the ratio of the total particle mass injected in the computational domain to the size of sample particles. By counting positions of each particle, the calculated particle mass concentrations $C$ in each room can be determined by

$C=\frac{M_{\mathrm{S}}}{V}$,

where $M_{\mathrm{S}}$ is the total mass of suspended particles in each room (or zone), and $V$ is the volume of each room.

\subsection{Validation of Lagrangian particle-tracking model}

To validate the accuracy of the Lagrangian particletracking model developed herein, model verification is firstly conducted to ensure the precision of the model by the reliable measured indoor mass concentrations of a fullscale two-zone building [11]. The experimental chamber



(b)
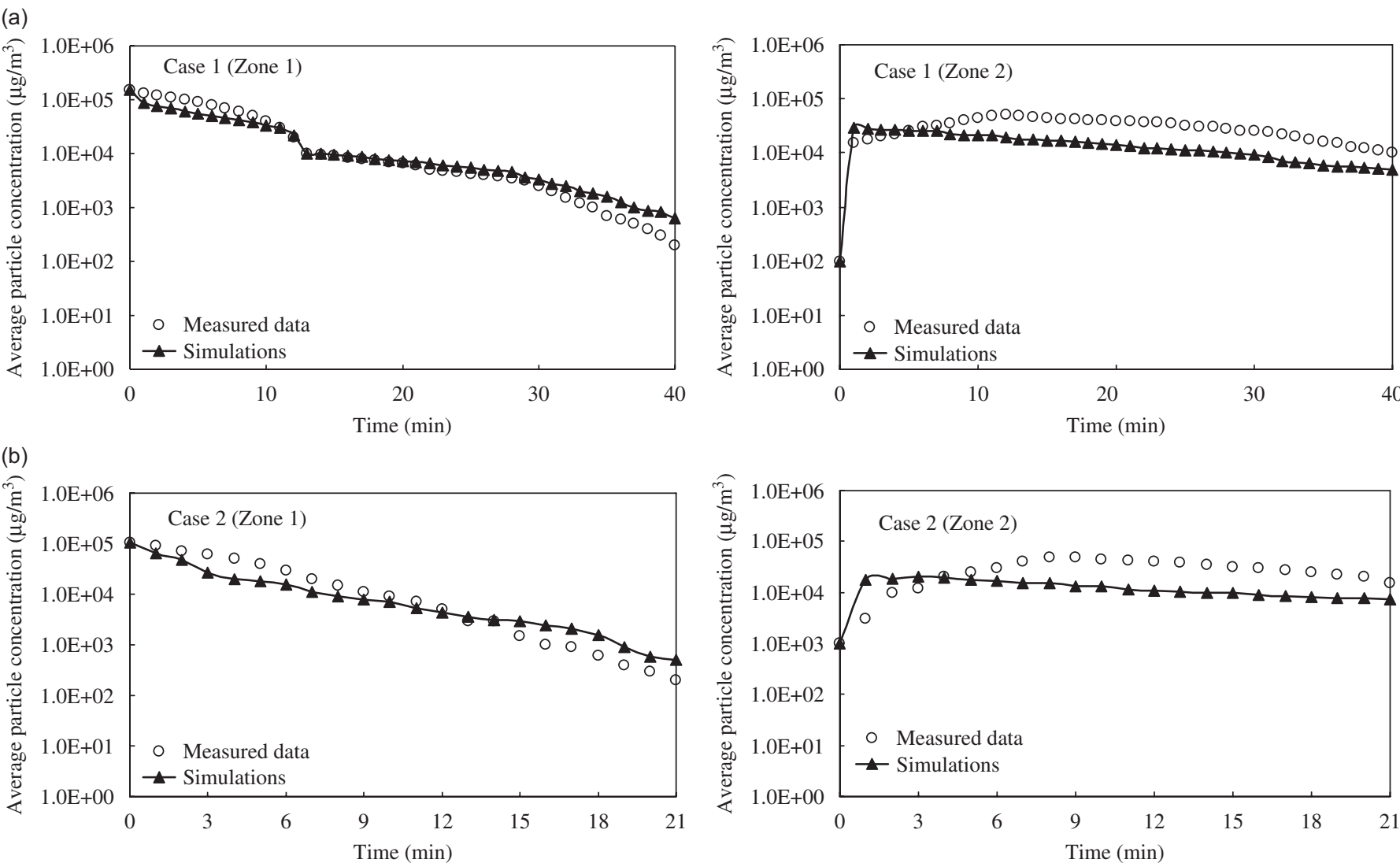

(10)

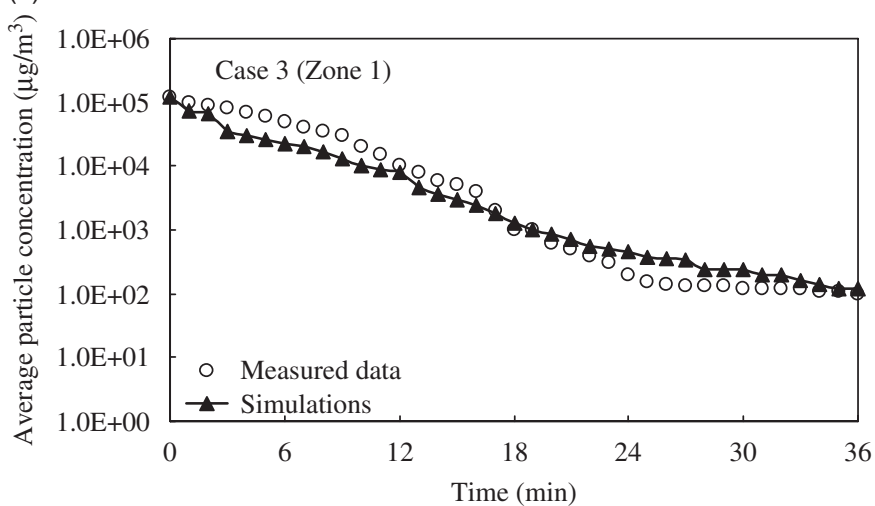

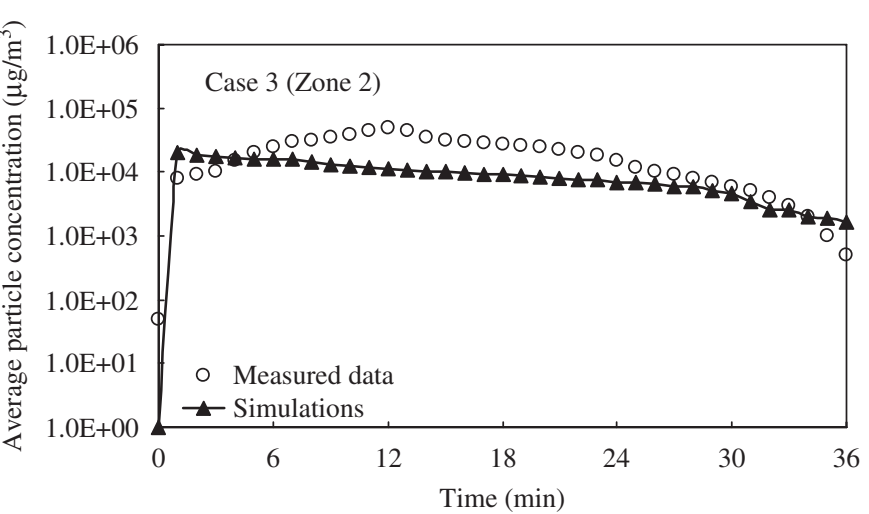

Fig. 2. Comparison of the simulated particle mass concentrations with the measured data conducted by $\mathrm{Lu}$ et al. (1999). (a) $\mathrm{ACH}=11.48 \mathrm{~h}^{-1}$, (b) $\mathrm{ACH}=14.00 \mathrm{~h}^{-1}$, (c) $\mathrm{ACH}=11.57 \mathrm{~h}^{-1}$, (d) $\mathrm{ACH}=12.71 \mathrm{~h}^{-1}$ (e) $\mathrm{ACH}=9.22 \mathrm{~h}^{-1}$, and (f) $\mathrm{ACH}=10.26 \mathrm{~h}^{-1}$. 
(d)

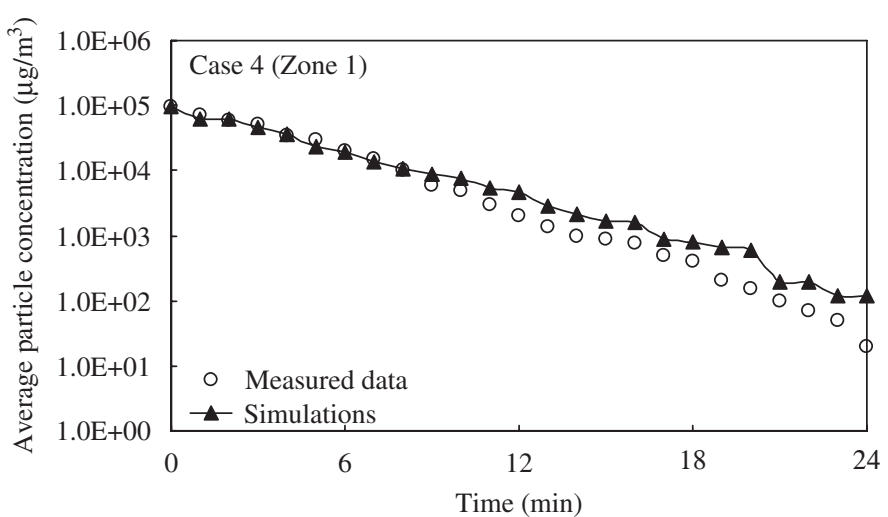

(e)

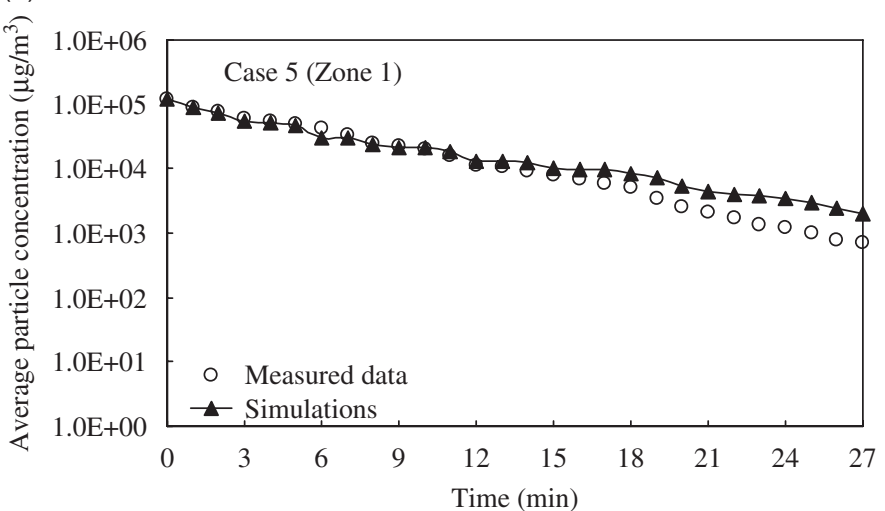

(f)

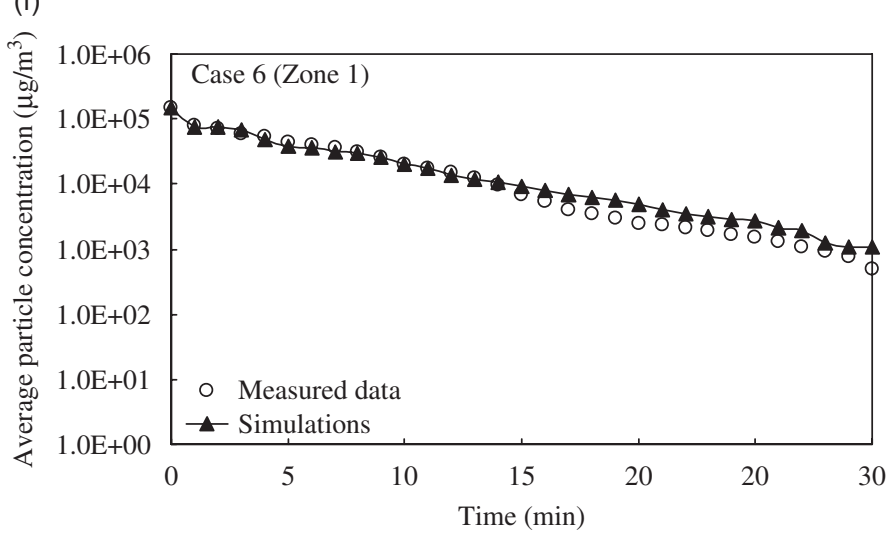


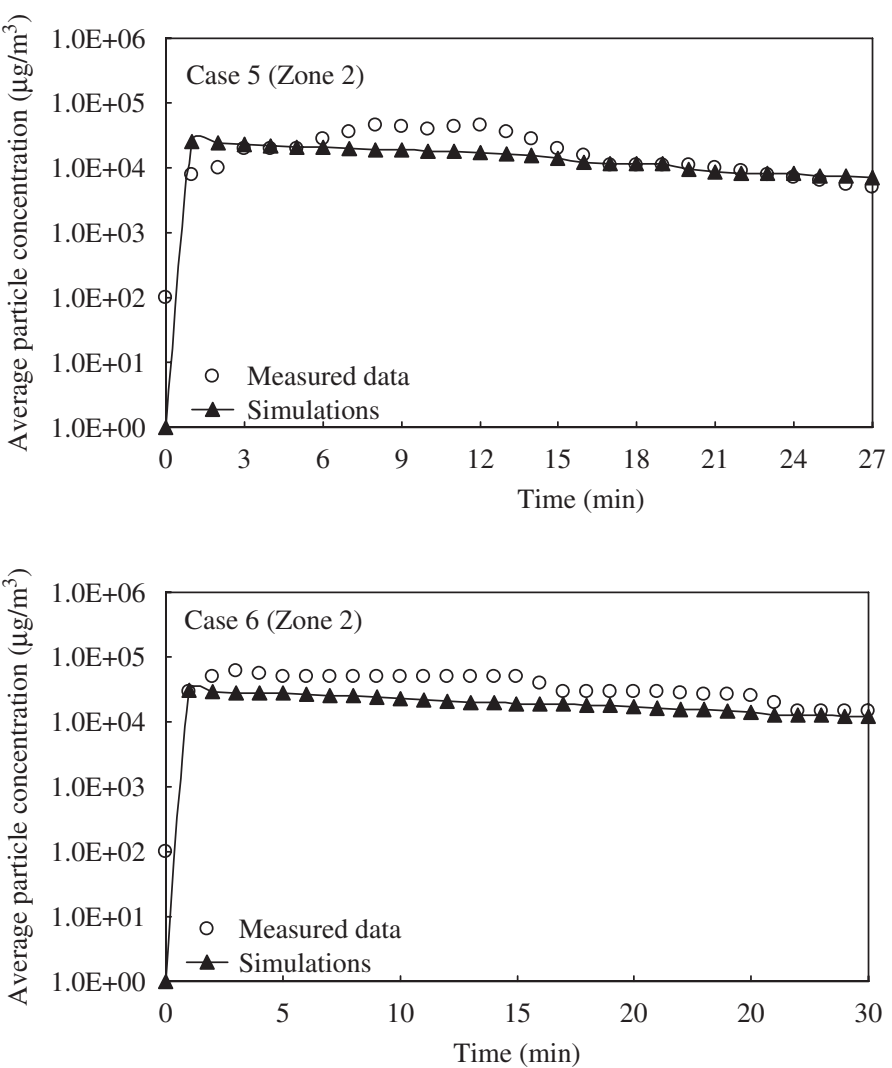

Fig. 2. (Continued)

used by $\mathrm{Lu}$ et al. has a size of $5.0 \mathrm{~m}$ in length, $3.0 \mathrm{~m}$ in width, and $2.4 \mathrm{~m}$ in height, as shown in Fig. 1. The chamber is divided into two same-size zones by a large partition with an opening. The interior opening is in the centerline of the chamber. There is an inflow vent $(1 \mathrm{~m} \times 0.5 \mathrm{~m})$ at higher location and an outlet vent $(1 \mathrm{~m} \times 0.5 \mathrm{~m})$ at lower location on two sides of this chamber separately. All of the walls and openings of the building are $0.15 \mathrm{~m}$ thick and have the same temperature as the ambient air. Constant airflow is supplied from the inflow. The airflow in the building is $20^{\circ} \mathrm{C}$ isothermal.

The released particles are equally divided into five size groups from 1 to $5 \mu \mathrm{m}$. The density of particles is $865.0 \mathrm{~kg} / \mathrm{m}^{3}$. The released particles are uniformly distributed in Zone 1 and motionless initially. The indoor mass concentrations of both zones are measured. Six cases have been conducted in their experiments, representing six air change rates $(\mathrm{ACH})$ with three sizes of interior openings (see Table 1). All of the six cases are used to verify the present numerical model. The relevant parameters used in the experiment are also listed in Table 1. Comparisons of the PM mass concentrations in both zones between the numerical solutions of the particle dynamic equation and the experiments are shown in Fig. 2. The mass concentration in Zone 1 lowers down rapidly because of particle deposition and migration to Zone 2. On account of the particles migrated from Zone 1, the mass concentration in Zone 2 for each case apparently increases in the first minute and then decreases with time. 
The agreement between the numerical simulations and the experiments for all the six cases is quite satisfactory.

\section{Specifications of numerical scenario simulations}

The Lagrangian particle-tracking model (the particle dynamic equation) has been validated in the previous section by the reliable experiment conducted by $\mathrm{Lu}$ et al. [11]. Next, the same two-zone building (with $0.95 \mathrm{~m}$-height interior opening) is adopted to study the transport mechanisms of size-dependent airborne particulate matters in partitioned indoor environment. The $\mathrm{ACH}$ for each room is reduced to $5 \mathrm{~h}^{-1}$ by lowering the incoming velocity of the inflow vent, because natural or mechanical ventilation in indoor environment rarely exceeds $7 \mathrm{~h}^{-1}$ [3].

Four major particle driving mechanisms (the gravitational force, the drag force, the Brownian motion force and the Saffman lift force) are considered in the model. Five kinds of particle transport mechanism scenarios are performed, including the dynamic equation scenario (all considered), the Brownian-motion-neglected scenario, the drag-force-neglected scenario, the lift-force-neglected scenario, and the inertial-force-neglected scenario (neglecting both the drag force and lift force). Seven different particle sizes $(10,5,2.5,1,0.5,0.1$ and $0.05 \mu \mathrm{m}$ in aerodynamic diameter), ranging from coarse to ultra-fine particle ranges, are used to investigate the relationship between particle diameter and each transport mechanism scenario. During the particle-tracking period, the trajectories and velocities of particles are recorded and the particle concentrations are thus calculated at every time step.

It is important to note that, in the LES approach, the smallest mesh used herein is $0.05 \mathrm{~m}$, which is near the wall region. After carrying out the LES for several flow-through times to ensure that the final time-averaged results are independent of the initial conditions, the time-averaged velocities and turbulent statistics are collected over $150 \mathrm{~s}$ with a temporal resolution of $\Delta t=0.003 \mathrm{~s}$. For the Lagrangian particle tracking process, the time step that we use is one order smaller than the relaxation time of particles to ensure the numerical accuracy; 600 ensembles are undertaken to obtain the ensemble-averaged trajectories for each sample particle at each time step $[15,16]$.

\section{Results and discussions}

\subsection{PM transport patterns}

By means of the LES computation and statistical analysis, the instantaneous and time-average airflow velocities are determined. Figs. 3(a) and (b) depict the calculated timeaveraged velocity distributions on the $X-Z$ plane at $Y=1.5 \mathrm{~m}$ (the centerline plane) and the $X-Y$ plane at $Z=0.5 \mathrm{~m}$, respectively. It can be clearly seen from these figures that there is a strong incoming jet formed near the inlet vent in Zone 1. The indoor partition limits the incoming jet (the primary flow) to move vertically downward towards

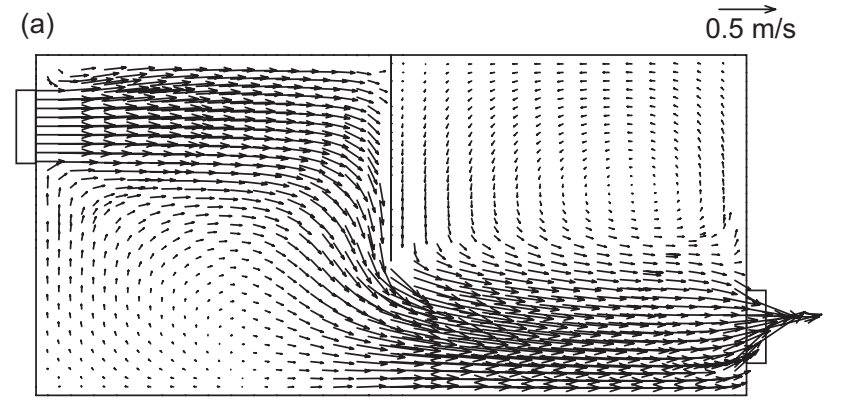

(b)

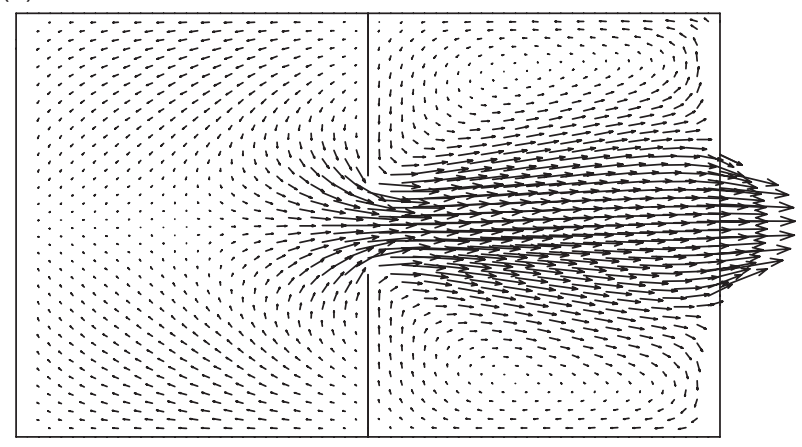

Fig. 3. The calculated time-averaged velocity distributions on (a) the $X-Z$ plane at $Y=1.5 \mathrm{~m}$, and (b) the $X-Y$ plane at $Z=0.5 \mathrm{~m}$.

the internal opening. A large clockwise-circulating region with weak velocity, occupying the lower part of Zone 1, is thus formed. In Zone 2, the primary airflow coming from the internal opening moves horizontally and finally exits at the outlet vent. A counterclockwise nonventilated circulation region, located at the upper part of Zone 2, is observed.

Particle trajectories calculated by the dynamic equation scenario for aerodynamic diameters of 10, 5, 2.5, 1, 0.5, 0.1, and $0.05 \mu \mathrm{m}$ are displayed in Figs. 4(a)-(g), respectively. To clearly demonstrate the effect of particle size on the particle trajectories, only 27 particles, uniformly released in Zone 1, are used for each aerodynamic diameter to plot Figs. 4(a)-(g). When a particle touches the trap boundary or passes through the outflow boundary, the particletracking process is terminated. It can be seen from Fig. 4 that for coarse particles $(\geqslant 2.5 \mu \mathrm{m})$, most of the particles are either expelled out of the indoor environment with the primary flow, or deposited onto the ground or walls. There is only a small number of particles trapped in the circulation regions. The particle-tracking process is thus short. On the other hand, for fine particles $(<2.5 \mu \mathrm{m})$, part of the particles follow the primary flow to flow out of the building, but many particles are trapped in the circulation regions with spiral-like paths. The particle-tracking process is thus long. As a result, it is observed that coarser particles are easier to be taken out of the building than fine particles. This phenomenon can be explained by the facts that coarse particles can maintain their velocities for longer time duration due to larger inertia and relaxation time. The above reason together with their stronger gravitational settling causes coarse particles to move easily from one circulation region to the other ones. On the contrary, fine 
(a)

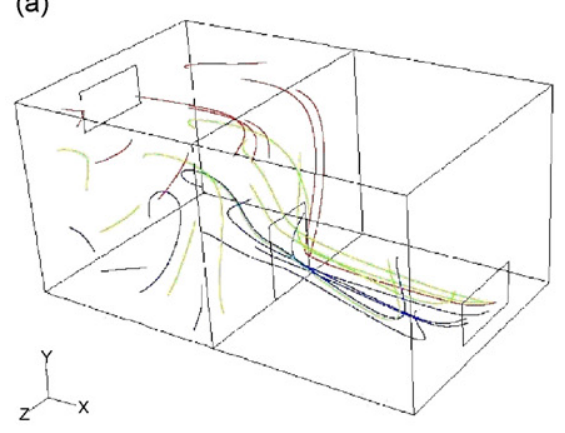

(c)

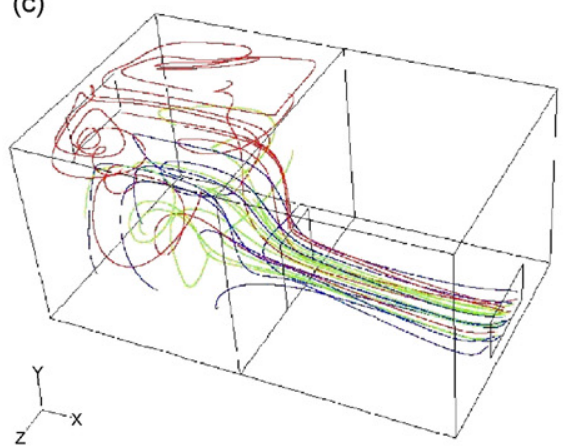

(e)

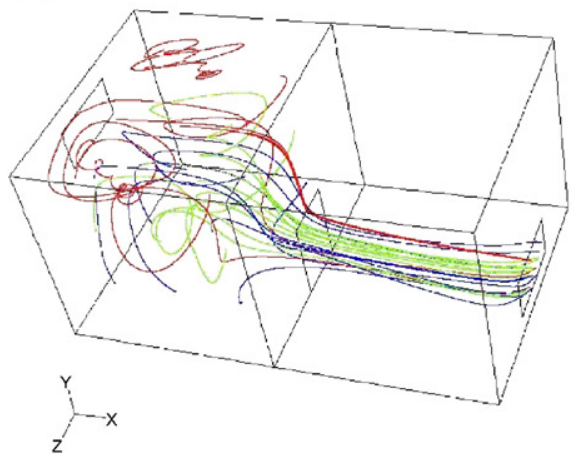

(b)

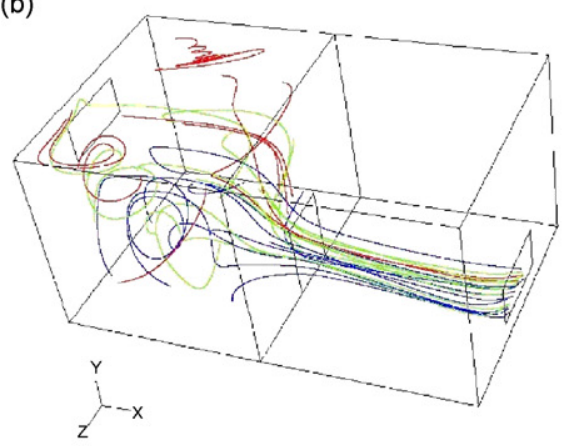

(d)

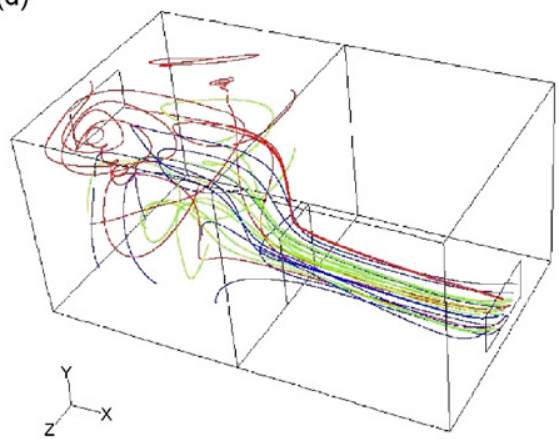

(f)

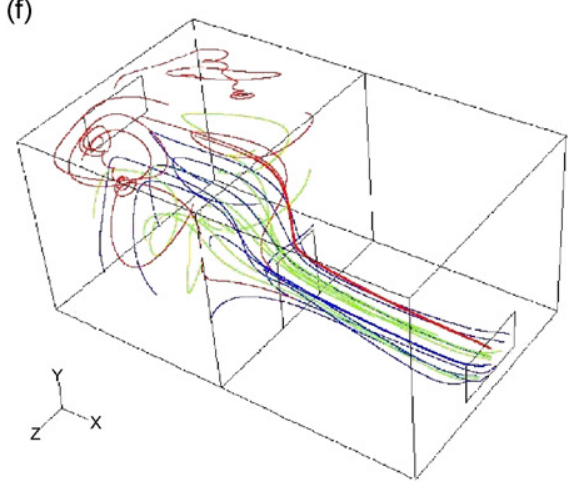

(g)

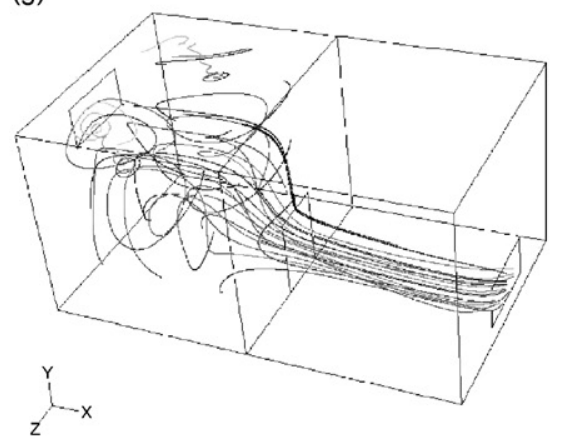

Fig. 4. Particle trajectories for various aerodynamic diameters, (a) 10, (b) 5, (c) 2.5, (d) 1, (e) 0.5, (f) 0.1, and (g) $0.05 \mu \mathrm{m}$.

particles possess smaller inertia and relaxation time, so they are easily influenced by the surrounding complex indoor airflow pattern of the two-zone building such as different length-scale vortex structures, entry momentum jets, and circulating flows, and trapped in eddies indoors. Thus, the indoor airflow pattern significantly affects the PM transport behaviors especially for fine particles.

\subsection{PM mass concentrations}

Based on the time variations of the particle trajectories in Fig. 4, the PM mass concentrations in Zone 1 and Zone 2 are next calculated by using Eq. (6). The calculated results of the PM mass concentrations for the Brownian-motionneglected scenario, the Saffman lift-force-neglected scenario, 
Table 2

Relative differences of the PM mass concentrations in Zone 1 and Zone 2 between the four scenarios and the dynamic equation scenario for each aerodynamic diameter at the 15 th minute of the tracking time

\begin{tabular}{|c|c|c|c|c|c|c|c|c|c|c|c|c|c|c|}
\hline \multirow{3}{*}{$\begin{array}{l}\text { Relative difference } \\
(\%)\end{array}$} & \multicolumn{14}{|c|}{ Particle aerodynamic diameter } \\
\hline & \multicolumn{2}{|l|}{$10 \mu \mathrm{m}$} & \multicolumn{2}{|l|}{$5 \mu \mathrm{m}$} & \multicolumn{2}{|l|}{$2.5 \mu \mathrm{m}$} & \multicolumn{2}{|l|}{$1 \mu \mathrm{m}$} & \multicolumn{2}{|l|}{$0.5 \mu \mathrm{m}$} & \multicolumn{2}{|l|}{$0.1 \mu \mathrm{m}$} & \multicolumn{2}{|l|}{$0.05 \mu \mathrm{m}$} \\
\hline & Zone 1 & Zone 2 & Zone 1 & Zone 2 & Zone 1 & Zone 2 & Zone 1 & Zone 2 & Zone 1 & Zone 2 & Zone 1 & Zone 2 & Zone 1 & Zone 2 \\
\hline Brownian motion force neglected & 0.14 & 0.74 & 1.31 & 2.67 & 1.08 & 3.14 & 3.41 & 4.56 & 3.91 & 5.96 & 4.48 & 8.20 & 8.53 & 15.31 \\
\hline Saffman lift force neglected & 2.13 & 0.14 & 6.62 & 17.32 & 3.82 & 6.50 & 3.40 & 5.02 & 1.22 & 4.17 & 0.70 & 1.06 & 0.19 & 0.24 \\
\hline Drag force neglected & 86.36 & 82.69 & 34.68 & 57.42 & 11.16 & 22.82 & 1.65 & 5.32 & 2.8 & 4.91 & 1.06 & 1.60 & 0.14 & 0.24 \\
\hline Inertial force neglected & 87.95 & 84.5 & 34.14 & 62.46 & 14.62 & 27.29 & 3.61 & 8.43 & 2.84 & 6.01 & 0.96 & 2.03 & 0.27 & 0.34 \\
\hline
\end{tabular}

the drag-force-neglected scenario, and the inertial-forceneglected scenario are compared with the results of the dynamic equation scenario. Table 2 shows the relative differences of the PM mass concentrations in Zone 1 and Zone 2 between the above four scenarios and the dynamic equation scenario for each aerodynamic diameter at the 15 th minute of the tracking time.

In Table 2, the importance of the Brownian motion force is increased as the particle aerodynamic diameter decreases. For particle size smaller than $0.5 \mu \mathrm{m}$, ignoring the Brownian motion force would result in apparent errors. The Saffman lift force on PM concentrations is apparently important between 2.5 and $5 \mu \mathrm{m}$. The neglect of the Saffman lift force for $5 \mu \mathrm{m}$ particle causes $17.32 \%$ in Zone 2. Contrary to the Brownian motion force, the significance of the drag force is increased as the particle aerodynamic diameter increases. The influence of the drag force becomes apparent for particle diameter larger than $2.5 \mu \mathrm{m}$, resulting in more than $15 \%$ averaged error for the entire building. As to the inertial-forceneglected scenario, neglecting both the drag force and the Saffman lift force gives more error than the drag-forceneglected scenario in PM mass concentration estimation. The inertial force is not negligible for particles larger than $1 \mu \mathrm{m}$.

\subsection{Particle trajectories}

After analyzing the influence of each driving mechanism on the PM mass concentrations, the impact of each driving mechanism on the PM trajectories is studied as well. In the Lagrangian particle tracking model, the positions and velocities of particles can be tracked at every time step. Based on the PM positions during the particle-tracking period, the calculated PM average deviations of displacement for the Brownian-motion-neglected scenario, the Saffman liftforce-neglected scenario, the drag-force-neglected scenario, and the inertial-force-neglected scenario are compared with that of the dynamic equation scenario. The average deviation of particle displacement is defined in the following:

$\Delta S(t)=\frac{\sum_{i=1}^{N} \sqrt{\left(x_{i}(t)-x_{\mathrm{dyn}, i}(t)\right)^{2}+\left(y_{i}(t)-y_{\mathrm{dyn}, i}(t)\right)^{2}+\left(z_{i}(t)-z_{\mathrm{dyn}, i}(t)\right)^{2}}}{N}$, where $N$ is the number of the released particles, $\left(x_{\mathrm{dyn}, i}(t)\right.$, $\left.y_{\mathrm{dyn}, i}(t), z_{\mathrm{dyn}, i}(t)\right)$ are the coordinates of particles calculated by the dynamic equation scenario at each particle-tracking time, and $\left(x_{i}(t), y_{i}(t), z i(t)\right)$ are the coordinates of particles for the other scenarios at each particle-tracking time.

Fig. 5 shows the average deviations of displacement during the tracking period for various aerodynamic diameters. For $10 \mu \mathrm{m}$ particles, as shown in Fig. 5(a), the average deviation of displacement is about $1 \mathrm{~m}$ for the drag-force-neglected scenario and the inertial-force-neglected scenario, and is about $0.01 \mathrm{~m}$ for the Saffman lift-force-neglected scenario. In addition, it is evident that the Brownian motion force makes very slight influence on the movement of $10 \mu \mathrm{m}$ particles (two orders smaller than the drag-force-neglected scenario). In Fig. 5(b), the drag-force-neglected scenario, the inertialforce-neglected scenario and the Saffman lift-forceneglected scenario have almost identical importance on particle trajectories for $5 \mu \mathrm{m}$ particles. Nevertheless, the influence of the Brownian motion force on $5 \mu \mathrm{m}$ particles can be still neglected compared to the other driving mechanisms.

For $2.5 \mu \mathrm{m}$ particles, the drag force still makes great impact on the movement of particles. However, as shown in Fig. 5(c), the Saffman lift force is less important compared to that for $5 \mu \mathrm{m}$ particles. In the case of $1 \mu \mathrm{m}$ particles (Fig. 5(d)), the drag-force-neglected scenario and the inertial-force-neglected scenario lead to the highest deviation. This means that the drag force is still not negligible. Meanwhile, the Saffman lift force has lesser influence, which is only as important as the Brownian motion force. For $0.5 \mu \mathrm{m}$ particles, the four scenarios induce the same deviation (Fig. 5(e)). In Figs. 5(f) and (g), the Brownian motion force is the most important force among the other driving forces. The average deviation of displacement caused by neglecting the Brownian motion force is nearly 10 times greater than that caused by neglecting the drag force for 0.1 and $0.05 \mu \mathrm{m}$ particles. Hence, the Brownian motion force has to be considered for ultra-fine and nano-scale particles.

It is important to note that the effect of the Saffman lift force on the average deviation of displacement becomes more significant in a specific range of particle sizes between 
(a)

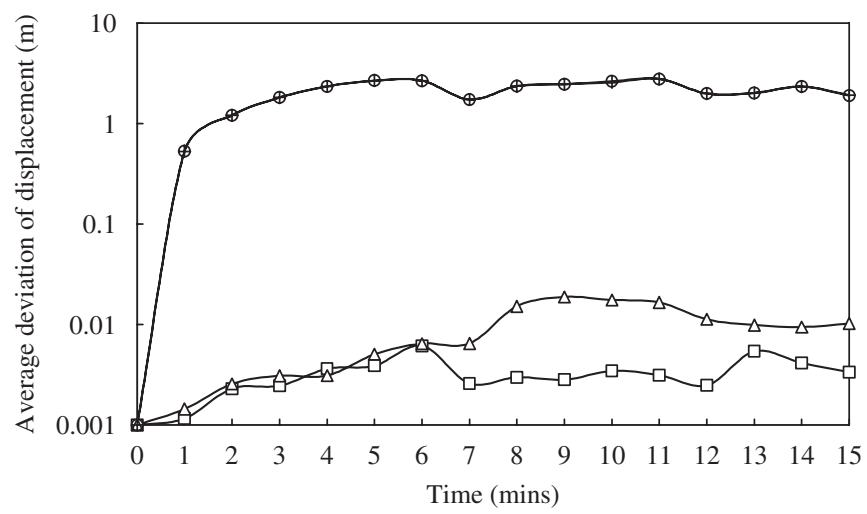

(c)

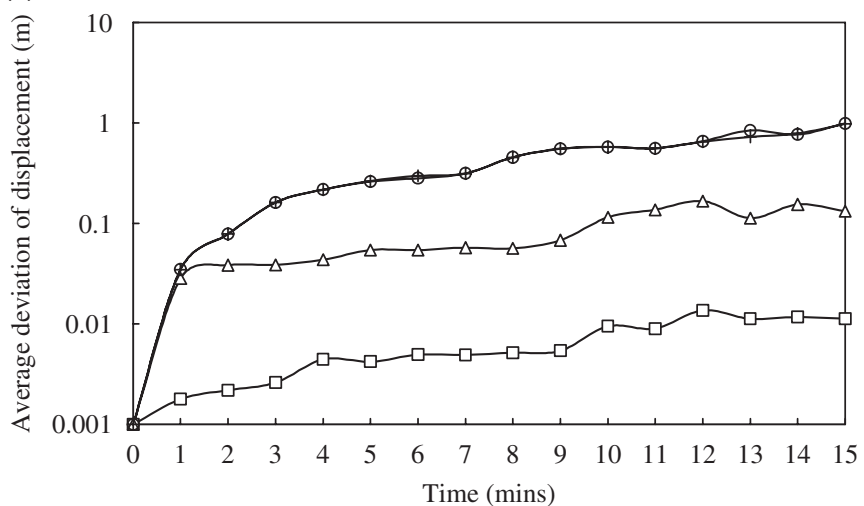

(e)

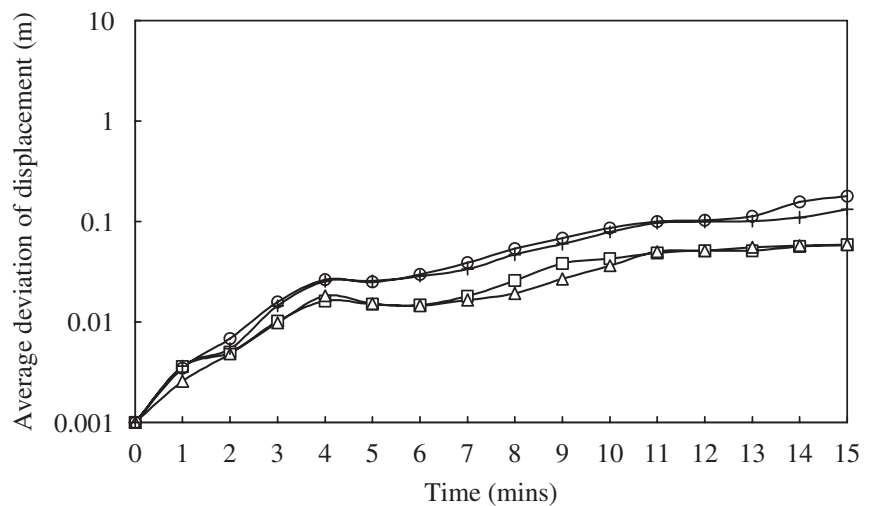

(b)

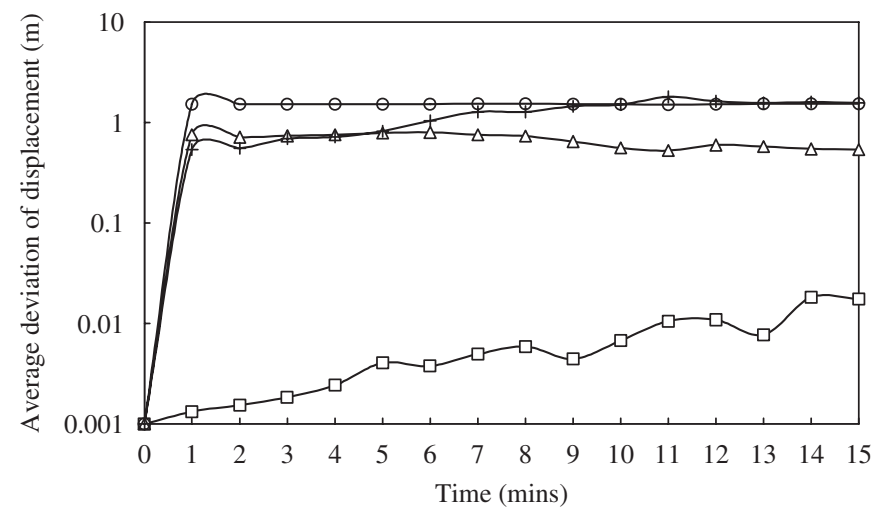

(d)

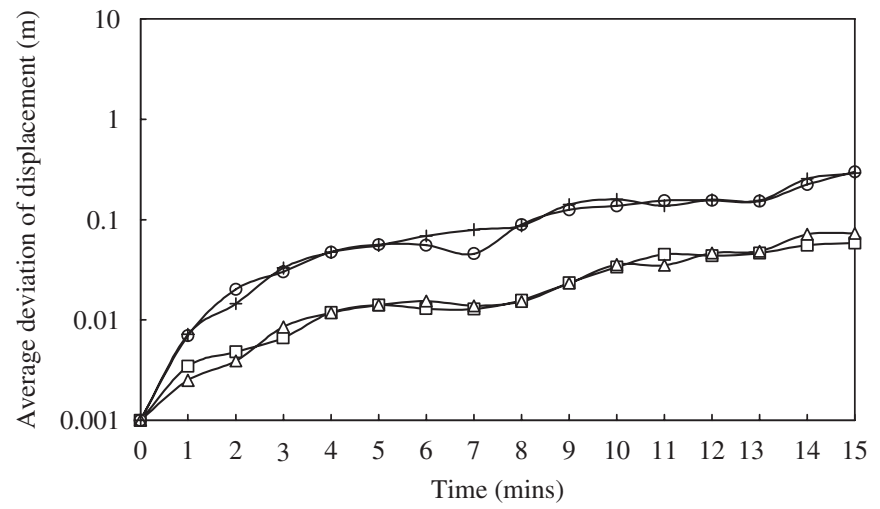

(f)

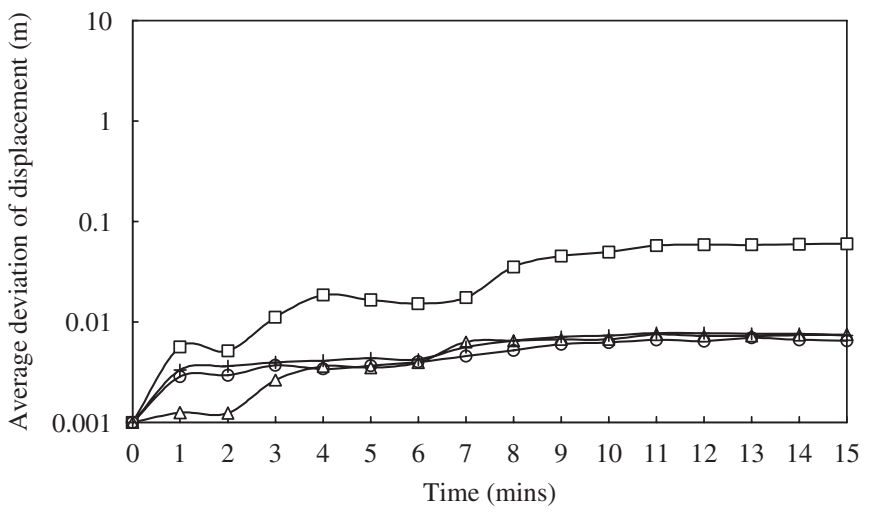

(g)

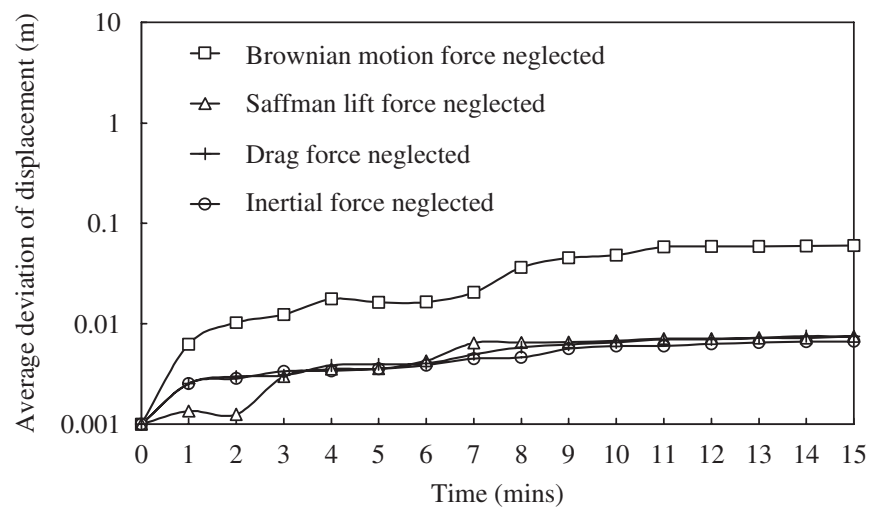

Fig. 5. The average deviations of particle displacement during the tracking period for various aerodynamic diameters, (a) 10, (b) 5, (c) 2.5, (d) 1, (e) 0.5, (f) 0.1 , and (g) $0.05 \mu \mathrm{m}$. 
2.5 and $5 \mu \mathrm{m}$. For particle diameter above or below this specific range, the Saffman lift force is negligible.

\section{Conclusions}

Based on the simulated results, the present research has led to the following conclusions:

(1) For coarse particles, most of the particles are either expelled out of the indoor environment with the primary flow, or deposited onto the ground or wall. The particle-tracking process is short. This is because coarse particles can maintain their velocities for longer time duration due to larger inertia and relaxation time, so that coarse particles can move easily from one circulation region to the other ones.

(2) For fine particles, part of the particles follow the primary flow to flow out of the building, but many particles are trapped in the circulation regions with spiral-like paths. The particle-tracking process is thus long. This can be explained by the fact that fine particles possess smaller inertia and relaxation time, so they are easily influenced by the surrounding complex indoor airflow pattern of the two-zone building and trapped in eddies indoors.

(3) According to the calculated PM mass concentrations for various aerodynamic diameters, the significance of the drag force is increased as the particle diameter increases, whereas the importance of the Brownian motion force is increased as the particle diameter decreases. The influence of the drag force becomes apparent for particle diameter larger than $2.5 \mu \mathrm{m}$. For particle size smaller than $0.5 \mu \mathrm{m}$, ignoring the Brownian motion force would result in apparent errors. The Saffman lift force on PM concentrations is apparently important between 2.5 and $5 \mu \mathrm{m}$. As to the inertialforce-neglected scenario, the inertial force is not negligible for particles larger than $1 \mu \mathrm{m}$.

(4) Based on the calculated PM trajectories for various aerodynamic diameters, the drag force and the inertia force make great impact on particle movement for particles larger than $1 \mu \mathrm{m}$. On the contrary, the Brownian motion force is more important for particles smaller than $0.5 \mu \mathrm{m}$. The Saffman lift force becomes more significant in a specific range of particle sizes between 2.5 and $5 \mu \mathrm{m}$. For particle diameter above or below this specific range, the Saffman lift force is negligible.

\section{References}

[1] Ostro BD. Associations between morbidity and alternative measures of particulate matter. Risk Analysis 1990;10:421-7.

[2] Dockery DW, Pope CA. Acute respiratory effects of particulate air pollution. Annual Reviews of Public Health 1994;15:107-32.

[3] Awbi HB. Ventilation of building. London: Chapman \& Hall; 1991.

[4] Hinds WC. Aerosol technology: properties, behavior, and measurement of airborne particles. 2nd ed. New York: Wiley-Interscience; 1999.

[5] Ekberg LE. Relationship between indoor and outdoor contaminants in mechanically ventilated buildings. Indoor Air 1996;6:41-7.

[6] Chang TJ. Numerical evaluation of the effect of traffic pollutant on indoor air quality of a naturally ventilated building. Journal of the Air and Waste Management Association 2002;52:1043-53.

[7] Chang TJ, Huang MY, Wu YT, Liao CM. Quantitative prediction of traffic pollutant transmission into buildings. Journal of Environmental Science and Health Part A 2003;A38(6):1025-40.

[8] Zhao B, Zhang Z, Li X, Huang D. Comparison of diffusion characteristics of aerosol particles in different ventilated rooms by numerical method. ASHRAE Transactions 2004;110:88-95.

[9] Lu W, Howarth AT. Numerical analysis of indoor aerosol particle deposition and distribution in two-zone ventilation system. Building and Environment 1996;31:41-50.

[10] Lu W, Howarth AT, Adam NM, Riffat SB. Modeling and measurement of airflow and aerosol particle distribution in a ventilated two-zone chamber. Building and Environment 1996;31: $417-23$.

[11] Lu W, Howarth AT, Adam NM, Riffat SB. CFD modeling and measurement of aerosol particle distributions in ventilated multizone rooms. ASHRAE Transactions 1999;105:116-27.

[12] Beghein C, Jiang Y, Chen QY. Using large eddy simulation to study particle motions in a room. Indoor Air 2005;15:281-90.

[13] Chung KC. Three-dimensional analysis of airflow and contaminant particle transport in a partitioned enclosure. Building and Environment 1999;34:7-17.

[14] Zhao B, Zhang Y, Li X, Yang X, Huang D. Comparison of indoor aerosol particle concentration and deposition in different ventilated rooms by numerical method. Building and Environment 2004;39:1-8.

[15] Chang TJ, Hsieh YF, Kao HM. Numerical investigation of airflow pattern and particulate matter transport in naturally ventilated multiroom buildings. Indoor Air 2006;16:136-52.

[16] Chang TJ, Kao HM, Hsieh YF. Numerical study of the effect of ventilation pattern on coarse, fine, and very fine particulate matter removal in partitioned indoor environment. Journal of the Air and Waste Management Association, 2007; 57:179-89.

[17] Li A, Ahmadi G. Deposition of aerosols on surfaces in a turbulent channel flow. International Journal of Engineering Science 1993;31:435-51.

[18] Chang TJ, Yen BC. Gravitational fall velocity of sphere in viscous fluid. Journal of Engineering Mechanics, ASCE 1998;124: 1193-9.

[19] Ahmadi G, Li A. Computer simulation of particle transport and deposition near a small isolated building. Journal of Wind Engineering and Industrial Aerodynamics 2000;84:23-46.

[20] Rao SS. Applied numerical methods for engineers and scientists. New Jersey: Prentice-Hall; 2002. 